\title{
Analytical BER Analysis of the V-BLAST in a Rayleigh Fading Channel
}

\author{
Sergey Loyka \\ School of Information Technology and Engineering, \\ University of Ottawa, \\ 161 Louis Pasteur, Ottawa, Ontario, Canada, K1N 6N5 \\ E-mail: sergey.loyka@ieee.org
}

\author{
Francois Gagnon \\ Department of Electrical Engineering \\ Ecole de Technologie Superieure \\ 1100, Notre-Dame St. West, Montreal \\ Quebec, H3C 1K3, Canada \\ E-mail: francois.gagnon@etsmtl.ca
}

\begin{abstract}
The BLAST algorithm is simple and, hence, popular solution for a signal processing at the MIMO receiver. In this paper, we present a closed-form analytical analysis of the V-BLAST without optimal ordering. A result on the zero-forcing maximum ratio combining weights at each detection step is derived to obtain a number of results: independence of noise, distribution of signal to noise ratio and block or bit error rates. We present a detailed analytical analysis and closed-form expressions for instantaneous and average BER at each detection step, which account for the error propagation and hold true for any modulation format and take simple form in some cases (BPSK). Asymptotic form, for large average $\mathrm{SNR}$, of these expressions is especially simple; the effect of the error propagation in this mode is to increase the total average BER by about 20, which is not catastrophic at all. It is demonstrated that the conventional V-BLAST and QR-decomposition based V-BLAST are essentially identical and have the same performance. Extensive Monte-Carlo simulations validate the analytical results and conclusions.
\end{abstract}

Index Terms-MIMO, V-BLAST, multi-antenna system, BER, outage, fading,

\section{INTRODUCTION}

The BLAST algorithm is simple and, hence, popular solution for a signal processing at the MIMO receiver, which also achieves the full MIMO capacity [1-3]. Its BER performance has been studied mainly numerically (Monte-Carlo techniques) since analytical analysis presents serious difficulties, especially when no bounds or approximations are used. While $2 \times n$ system (i.e. with $2 \mathrm{Tx}$ and $\mathrm{n} \mathrm{Rx}$ antennas) can be analytically analyzed in a closed form without using any bounds [4,5], an extension of the analysis to the general case of mxn system has not been found yet. Hence various bounds and approximations have been employed to attack the problem [6]. Consequently, the solutions found are limited in some way. A limitation of $[4,5]$ is that a non-coherent equal gain combining (NC EGC) was used after interference nulling out (projection), which is not optimum, and that the after-projection noise correlation was ignored.

The approach adopted in the present paper is different. Close examination of the problem of BLAST BER performance analysis reveals that the major difficulty for closed-from exact analytical analysis is due to the optimal ordering procedure. Hence, we analyze the algorithm performance without optimal ordering. Clearly, this is a disadvantage of the analysis. However, there are certain advantages as well: (i) closed-form exact analytical analysis is possible in the general case of mxn system, which results in exact BER expressions, (ii) this provides deep insight and understanding that cannot be gained using Monte-Carlo approach alone, (iii) there exists a hope that the techniques developed can be further extended to account for the optimal ordering, (iv) comparing the performance of the no-ordering algorithm to that with the optimal ordering allows one to better understand the advantages provided by the ordering and various differences in the performance, (v) computational complexity of the algorithm without optimal ordering is significantly less and, hence, an implementation complexity is smaller. Contrary to [5], we employ the maximum ratio combining (MRC) after the interference projection (taking into account the after-projection noise correlation), which provides the best performance in terms of output SNR and, hence, BER. This also results in exact closed-form expressions for unconditional average BER at each step, which is not available in [5], or, to the best of our knowledge, elsewhere.

The approach consists essentially in obtaining new results on the optimal MRC weights without ordering. Theses weights are expressed as a product of a projection matrix by part of the channel matrix and it is thus possible to prove that the aftercombining noise and inter-stream interference (ISI) at each detection step are Gaussian and independent. A closed form expression of the output SNR distributions at each step is obtained with an assumption of error-free detection at the previous steps. These distributions are used to obtain relatively precise outage, block, bit and total bit error rates. A more detailed analysis, which still makes good use of the MRC weight properties but with conditioning on previous step error events, permits to obtain exact BER expressions for BPSK modulation without the error-free detection assumption, i.e. taking into account the error propagation. Finally, by analyzing the details of the QR decomposition and V-BLAST, it is demonstrated that the MRC weight matrix and the $\mathrm{Q}$ matrix in QR decomposition are equal and, hence, performance of the regular V-BLAST and the QR- based BLAST are the same.

\section{THE V-BLAST ALgORITHM}

The main idea of the V-BLAST is to split the information bit stream into several sub-streams and transmit them in parallel using a set of transmit (Tx) antennas at the same time and frequency. At the receiver (Rx) end, each Rx antennas "sees" all the transmitted signals, which are mixed due to the nature 
of the wireless propagation channel. Using appropriate signal processing at the $\mathrm{Rx}$, these signals can be unmixed so that the matrix wireless channel is transformed into a set of virtual parallel independent channels (provided that the multipath is rich enough).

The standard baseband system model is given by

$$
\mathbf{r}=\mathbf{H s}+\xi
$$

where $\mathbf{s}$ and $\mathbf{r}$ are the Tx and Rx vectors correspondingly, $\mathbf{H}$ is the mxn channel matrix, i.e. the matrix of the complex channel gains between each Tx and each $\mathrm{Rx}$ antenna, $\mathrm{n}$ is the number of $\mathrm{Rx}$ antennas, $\mathrm{m}$ is the number of $\mathrm{Tx}$ antennas, $n \geq m$, and $\xi$ is the additive white Gaussian noise (AWGN), which is assumed to be $\mathcal{C N}\left(0, \sigma_{0}^{2} \mathbf{I}\right)$, i.e. independent and identically distributed (i.i.d.) in each branch.

The job of the V-BLAST algorithm is to find $\mathbf{s}$ given $\mathbf{r}$ and $\mathbf{H}$ in a computationally-efficient way. The V-BLAST processing begins with the $1^{\text {st }} \mathrm{Tx}$ symbol and proceeds in sequence to the $\mathrm{m}$-th symbol. When the optimal ordering procedure is employed, the Tx indexing is changed prior to the processing. The main steps of the algorithm are as follows $[1,3]$ :

(1) The interference cancellation step: at the i-th processing step (i.e., when the signal from the i-th transmitter is detected) the interference from the first $(i-1)$ transmitters can be subtracted based on the estimations of the Tx symbols and the knowledge of the channel matrix $\mathbf{H}$,

$$
\mathbf{r}_{i}^{\prime}=\mathbf{r}-\sum_{j=1}^{i-1} \mathbf{h}_{j} \hat{s}_{j}
$$

where $\mathbf{h}_{j}$ is the $\mathbf{j}$-th column of $\mathbf{H}$, and $\hat{s}_{j}$ are the detected symbols (which are assumed to be error-free).

(2) The interference nulling step: based on the knowledge of the channel matrix, the interference from yet-to-be-detected symbols can be nulled out using the Gram-Schmidt orthogonalization process (applied to the column vectors of $\mathbf{H}$ ) and orthogonal projection on the sub-spaced spanned by yetto-be-detected symbols,

$$
\mathbf{r}_{i}^{\prime \prime}=\mathbf{P}_{i} \mathbf{r}_{i}^{\prime}
$$

where $\mathbf{P}_{i}$ is the projection matrix on the sub-space orthogonal to that spanned by $\left\{\mathbf{h}_{i+1} \mathbf{h}_{i+2} \ldots \mathbf{h}_{m}\right\}: \mathbf{P}_{i}=\mathbf{I}-\mathbf{H}_{i}\left(\mathbf{H}_{i}^{+} \mathbf{H}_{i}\right)^{-1} \mathbf{H}_{i}^{+}$, where $\mathbf{H}_{i}=\left[\mathbf{h}_{i+1} \mathbf{h}_{i+2} \ldots \mathbf{h}_{m}\right][8]$.

(3) The optimal ordering procedure: the order of symbol processing is organized according to their after-processing SNRs in the decreasing order, i.e. the symbol with highest SNR is detected first.

\section{ANALYSIS OF THE V-BLAST ALGORITHM}

The following basic assumptions are employed in the present paper:

(1) The channel is random, quasistatic (i.e. fixed for every frame of information bits but varying from frame to frame), frequency independent (i.e., negligible delay spread); the components of $\mathbf{H}$ are $\mathcal{C N}(0, \mathbf{I})$ (i.e., i.i.d. Rayleigh fading with unit average power gain).

(2) Equal-power constellations are used.

(3) The Tx signals, noise and channel gains are independent of each other.

(4) Perfect channel knowledge is available at the receiver, but not at the transmitter.

(5) There is no performance degradation due to synchronization and timing errors.

It should be noted that some of the results below do not need all these assumptions. As it was indicated above, the optimal ordering procedure will be omitted in the present paper. We follow the approach to V-BLAST analysis proposed in $[4,5]$, where it was shown that the conditional (i.e. assuming no detection error at the first $(i-1)$ steps) after-processing instantaneous (i.e. for given channel instant) signal power $P_{s i}$ at i-th processing step is

$$
P_{s i} \sim \chi_{2(n-m+i)}^{2}
$$

where $\sim$ means equal in distribution, and different $P_{s i}$ are independent of each other. The i-th step has diversity order equal to $(n-m+i)$, the smallest one being at the $1^{\text {st }}$ step and the largest - at the last one. Note that the fact that the distribution is conditional (no error propagation) does not limit the analysis since, as we show below, the conditional distribution at each step is sufficient to find the block error rate (BLER) and outage probability taking into account the error propagation. The distribution of $P_{s i}$ follows also from the Bartlett decomposition of the complex Wishart matrix [7].

The best way to improve the output SNR is to use maximum ratio combining (MRC) after the interference nulling out step, i.e. to form the decision variable

$$
\widehat{r_{i}}=\mathbf{w}_{i}^{+} \mathbf{r}_{i}^{\prime \prime}
$$

where $\mathbf{w}_{i}^{+}$are the optimum weights, and " "'" means Hermitian conjugate. However, the well-known MRC expressions for the weights and the output SNR cannot be applied directly since the orthogonal projection during the nulling-out step (see (3)) results in correlated branch noise. The after-projection noise correlation matrix is

$$
\mathbf{C}_{\xi_{i}}=\left\langle\mathbf{P}_{i} \xi \xi^{+} \mathbf{P}_{i}\right\rangle=\sigma_{0}^{2} \mathbf{P}_{i}
$$

where $<>$ denotes expectation (in this case, over the noise). The MRC combining weights $\mathbf{w}_{i}$ are given in this case by the solution of the following generalized eigenvalue problem,

$$
\left(\mathbf{C}_{s i}-\gamma_{i} \mathbf{C}_{\xi_{i}}\right) \mathbf{w}_{i}=0
$$

where $\mathbf{C}_{s i}$ is the after-projection signal covariance matrix at step $i$. As detailed analysis demonstrates (the proof is omitted here due to the page limits; more details are given in the extended journal version of this paper [13]), the optimum (MRC) weights are given by 


$$
\mathbf{w}_{i}=\mathbf{P}_{i} \mathbf{h}_{i},
$$

Since these weights already include the projection, they can be applied directly to $\mathbf{r}_{i}^{\prime}$ (i.e. these are zero-forcing MRC weights). The output signal can be presented as

$$
\hat{r_{i}}=\mathbf{w}_{i}^{+} \mathbf{r}_{i}^{\prime}=r_{s i}+r_{\xi i}, r_{s i}=\mathbf{w}_{i}^{+} \mathbf{h}_{i} s_{i}, r_{\xi_{i}}=\mathbf{w}_{i}^{+} \boldsymbol{\xi},
$$

Remarkably, the output SNR is still the same as that for an i.i.d. branch noise,

$$
\gamma_{i}=\sigma_{0}^{-2} P_{s i}=\sigma_{0}^{-2} \mathbf{y}_{i}^{+} \mathbf{y}_{i}=\sigma_{0}^{-2} \mathbf{h}_{i}^{+} \mathbf{P}_{i} \mathbf{h}_{i}
$$

where $\mathbf{y}_{i}=\mathbf{P}_{i} \mathbf{h}_{i} s_{i}$ is the after-projection signal and we have assumed, without loss of generality, that $\left|s_{i}\right|^{2}=1$ (i.e., equal power constellation). We stress that this is a non-trivial result that holds true because of special structure of the projection matrix $\mathbf{P}$.

Since $P_{s i}$ at different steps are independent of each other, so are $\gamma_{i}$. From this, however, it does not follow that the decisions and, hence, errors at each step are independent of each other as the latter requires for the noise $r_{\xi i}$ to be independent at each step, not just the SNR. It can be shown (based on the properties of the projection matrices and using (8)) that the optimum weights at different steps are orthogonal to each other,

$$
\mathbf{w}_{i}^{+} \mathbf{w}_{j}=\mathbf{h}_{i}^{+} \mathbf{P}_{i} \mathbf{P}_{j} \mathbf{h}_{j}=\mathbf{0} \forall i \neq j,
$$

Consider now the output noise covariance matrix,

$$
R_{i j}=\left\langle r_{\xi_{i}} r_{\xi_{j}}\right\rangle \text {, }
$$

where the expectation is over the noise. Using (8), (9) and (11), one obtains,

$$
R_{i j}=\sigma_{0}^{2} \mathbf{w}_{i}^{+} \mathbf{w}_{j}=\sigma_{0}^{2}\left|\mathbf{w}_{i}\right|^{2} \delta_{i j}
$$

where $\delta_{i j}=1$ if $i=j$ and 0 otherwise. Since $r_{\xi i}=\mathbf{w}_{i}^{+} \xi$ are complex Gaussian (for given channel realization), independence follows from zero correlation. Hence, the decisions and errors are independent at each step and the conditional error rates are independent too.

\section{EXACT BER EXPRESSIONS FOR BPSK MODULATION}

In this section, we derive exact closed-form expressions for step BER with BPSK modulation. We use the normalized weights $\left|\mathbf{w}_{i}\right|^{2}=1$ (note that normalization does not affect SNR and, hence, BER) so that the weight matrix $\mathbf{W}=\left[\mathbf{w}_{1}, \mathbf{w}_{2}, \ldots \mathbf{w}_{m}\right]$ is semi-unitary (it is unitary only when $m=n)$,

$$
\mathbf{W}^{+} \mathbf{W}=\mathbf{I}
$$

This normalization corresponds to $\mathbf{w}_{i}=\mathbf{P}_{i} \mathbf{h}_{i} /\left|\mathbf{P}_{i} \mathbf{h}_{i}\right|$. For simplicity, we begin with $2 \mathrm{x} n$ system. In our analysis, we follow a similar approach of multiuser detection BER analysis [11].

\section{A. 2xn V-BLAST BER}

The output signal at step 2 is

$$
r_{2}=\mathbf{w}_{2}^{+} \mathbf{h}_{2} s_{2}+\mathbf{w}_{2}^{+} \mathbf{h}_{1} \Delta s_{1}+\mathbf{w}_{2}^{+} \boldsymbol{\xi},
$$

where $\mathbf{w}_{2}=\mathbf{h}_{2} /\left|\mathbf{h}_{2}\right|$ and $\Delta s_{1}=s_{1}-\hat{s}_{1}$. Conditioned on $\mathbf{h}_{2}$ and $\Delta s_{1}$, the inter-stream interference (ISI) $z_{21}=\mathbf{w}_{2}^{+} \mathbf{h}_{1} \Delta s_{1}$ is Gaussian, $\left.z_{21}\right|_{\mathbf{h}_{2}, \Delta s_{1}} \sim C N\left(0,\left|\Delta s_{1}\right|^{2}\right)$. To see this, we first note that, for given $\mathbf{h}_{2}$ and $\Delta s_{1}, z_{21}$ is a sum of Gaussian random variables, and, hence, is Gaussian. The conditional mean and variance of $z_{21}$ are

$$
\left\langle z_{21}\right\rangle=0, \quad\left\langle\left|z_{21}\right|^{2}\right\rangle=\mathbf{w}_{2}^{+}\left\langle\mathbf{h}_{1} \mathbf{h}_{1}^{+}\right\rangle \mathbf{w}_{2}\left|\Delta s_{1}\right|^{2}=\left|\Delta s_{1}\right|^{2},
$$

as $\left\langle\mathbf{h}_{1}\right\rangle=\mathbf{0}$ and $\left\langle\mathbf{h}_{1} \mathbf{h}_{1}^{+}\right\rangle=\mathbf{I}$. Since the conditional distribution does not depend on $\mathbf{h}_{2}$, the conditioning on $\mathbf{h}_{2}$ can be dropped, $\left.z_{21}\right|_{\Delta s_{1}} \sim C N\left(0,\left|\Delta s_{1}\right|^{2}\right)$. In the same way, one can see that the noise term in (15) is also Gaussian and independent of $\mathbf{h}_{2}, \mathbf{w}_{2}^{+} \boldsymbol{\xi} \sim C N\left(0, \sigma_{0}^{2}\right)$, and it is also independent of the other terms. The last two terms can be joined to get the "total noise" term, which includes the ISI, and, conditioned on $\Delta s_{1}$, its distribution is

$$
\mathbf{w}_{2}^{+} \mathbf{h}_{1} \Delta s_{1}+\mathbf{w}_{2}^{+} \xi \sim C N\left(0, \sigma_{0}^{2}+\left|\Delta s_{1}\right|^{2}\right)
$$

Since instantaneous BERs at each step are independent of each other and $\left|\Delta s_{1}\right|=0$ (no error at step 1) with probability $1-Q\left(\sqrt{2 \gamma_{1}}\right)$ and $\left|\Delta s_{1}\right|=2$ (error at step 1) with probability $Q\left(\sqrt{2 \gamma_{1}}\right)$, for given $\mathbf{h}_{2}$ BER at step 2 can be immediately found from (15) as

$$
P_{u 2}\left(\mathbf{h}_{2}\right)=Q\left(\sqrt{2 \gamma_{2}}\right)\left(1-\bar{P}_{e 1}\right)+Q\left(\sqrt{\frac{2 \mathbf{h}_{2}^{+} \mathbf{h}_{2}}{\sigma_{0}^{2}+4}}\right) \bar{P}_{e 1},
$$

where $\bar{P}_{e 1}=\left\langle Q\left(\sqrt{2 \gamma_{1}}\right)\right\rangle_{\gamma_{1}}$ is the average BER at step 1 (which is the same as (n-1)-order MRC average BER) and, with the adopted normalization, $\gamma_{0}=1 / \sigma_{0}^{2}$ is the average SNR. $P_{u 2}\left(\mathbf{h}_{2}\right)$ is unconditional (including error propagation from step 1) BER at step 2 averaged over $\mathbf{h}_{1}$ but not $\mathbf{h}_{2}$ (it is neither average not instantaneous BER in traditional sense). Finally, the average unconditional BER at step 2 is

$$
\bar{P}_{u 2}=\left\langle P_{u 2}\left(\mathbf{h}_{2}\right)\right\rangle_{\mathbf{h}_{2}}=\bar{P}_{e 2}\left(1-\bar{P}_{e 1}\right)+\bar{P}_{21} \bar{P}_{e 1}
$$

where $\bar{P}_{e 2}=\left\langle Q\left(\sqrt{2 \gamma_{2}}\right)\right\rangle_{\gamma_{2}}$ is the conditional (on no error at $1^{\text {st }}$ step) average BER at step 2 (which is the same as $n$-th order $\mathrm{MRC}$ average BER), and

$$
\bar{P}_{21}=\left\langle Q\left(\sqrt{\frac{2 \mathbf{h}_{2}^{+} \mathbf{h}_{2}}{\sigma_{0}^{2}+4}}\right)\right\rangle_{\mathbf{h}_{2}}=\bar{P}_{e 2}\left(\frac{1}{\sigma_{0}^{2}+4}\right),
$$

is the average probability of error propagation. In the case of $2 \times 2$ system the expressions become especially simple,

$$
\bar{P}_{e 1}=(1-\mu) / 2, \bar{P}_{e 2}=(1-\mu)^{2}(2+\mu) / 4,
$$


where $\mu=\sqrt{\gamma_{0} /\left(1+\gamma_{0}\right)}$. For large average SNR, $\sigma_{0}^{2} \ll 1$,

$$
\bar{P}_{21}=\bar{P}_{2}\left(\frac{1}{\sigma_{0}^{2}+4}\right) \approx \frac{1}{5}, \bar{P}_{2} \approx \frac{3}{4 \gamma_{0}^{2}}, \bar{P}_{1} \approx \frac{1}{4 \gamma_{0}},
$$

and (19) simplifies to

$$
\bar{P}_{u 2} \approx \bar{P}_{e 2}+\bar{P}_{21} \bar{P}_{e 1} \approx \bar{P}_{e 1} / 5 \approx 1 /\left(20 \gamma_{0}\right),
$$

Note that the second term dominates in (19) as it is the $1^{\text {st }}$ order term (no diversity) while the $1^{\text {st }}$ term is the $2^{\text {nd }}$ order one $\left(2^{\text {nd }}\right.$ order diversity). Clearly, there is no diversity at the $2^{\text {nd }}$ step due to the error propagation. The total average BER (i.e. the BER in the single output stream, which includes all the individual sub-streams) can be found as

$$
\overline{P_{e t}}=\frac{1}{m} \sum_{i=1}^{m} \overline{P_{u i}}
$$

In the large SNR case, this reduces to

$$
\bar{P}_{e t} \approx \bar{P}_{e 1} / 2 \approx 1 / 8 \gamma_{0}
$$

as the first step BER is dominant. Additionally, block error rate (BLER) can be defined as a probability of having at least one error at the Tx vector, which is also a useful performance measure. The average BLER is

$$
\bar{P}_{B}=\bar{P}_{e 2}\left(1-\bar{P}_{e 1}\right)+\bar{P}_{e 1},
$$

which can be further approximated, for high average SNR, as

$$
\bar{P}_{B} \approx \bar{P}_{e 1} \approx 1 /\left(4 \gamma_{0}\right),
$$

i.e. the first step average BER dominates in the average BLER, which provides a quick way to estimate the latter.

\section{B. nxm V-BLAST BER}

The derivation in this case follows the same steps as above and is omitted here due to the page limits. The results are as follows. The conditional BER at step $i$ averaged over $\left\{\mathbf{h}_{1}, \mathbf{h}_{2}, \ldots \mathbf{h}_{i-1}\right\}$ is

$$
P_{e, i \mid \mathbf{E}_{i-1}}=\operatorname{Pr}\left\{e_{i}=1 \mid \mathbf{E}_{i-1}\right\}=Q\left(\sqrt{\frac{2 \mathbf{h}_{i}^{+} \mathbf{P}_{i} \mathbf{h}_{i}}{\sigma_{0}^{2}+4\left|\mathbf{E}_{i-1}\right|^{2}}}\right),
$$

where $E_{i-1}=\left[e_{1}, e_{2} \ldots e_{i-1}\right]$ is the error vector: $e_{k}=1$ if there is an error at step $k$ and $e_{k}=0$ otherwise. $\operatorname{Pr}\left\{\mathbf{E}_{i-1}\right\}$ can be expressed as

$$
\operatorname{Pr}\left\{\mathbf{E}_{i-1}\right\}=\prod_{k=1}^{i-1} \operatorname{Pr}\left\{e_{k} \mid \mathbf{E}_{k-1}\right\},
$$

where

$$
\operatorname{Pr}\left\{e_{k} \mid \mathbf{E}_{k-1}\right\}=\left\{\begin{array}{l}
P_{e, k \mid \mathbf{E}_{k-1}}, e_{k}=1 \\
1-P_{e, k \mid \mathbf{E}_{k-1}}, e_{k}=0
\end{array}\right.
$$

The average unconditional BER at step $i$ is

$$
\bar{P}_{u, i}=\left\langle P_{u, i}(\mathbf{H})\right\rangle_{\mathbf{H}}=\sum_{\mathbf{E}_{i-1}} \bar{P}_{e, i \mid \mathbf{E}_{i-1}} \bar{P}\left[\mathbf{E}_{i-1}\right],
$$

where the summation is over all possible error patterns $\mathbf{E}_{i-1}$ and

$$
\begin{aligned}
& \bar{P}_{e, i \mid \mathbf{E}_{i-1}}=\left[\frac{1-\mu_{i}}{2}\right]^{n-m+i} \sum_{k=0}^{n-m+i-1} C_{n-m+i-1+k}^{k}\left[\frac{1+\mu_{i}}{2}\right]^{k}, \\
& \mu_{i}=\left(\sqrt{1+\sigma_{0}^{2}+4\left|\mathbf{E}_{i-1}\right|^{2}}\right)^{-1}, \bar{P}\left[\mathbf{E}_{i-1}\right]=\prod_{k=1}^{i-1}\left\langle\operatorname{Pr}\left\{e_{k} \mid \mathbf{E}_{k-1}\right\}\right\rangle
\end{aligned}
$$

$C_{n}^{k}=n ! /(k !(n-k) !)$ are the binomial coefficients, and $\left\langle\operatorname{Pr}\left\{e_{k} \mid \mathbf{E}_{k-1}\right\}\right\rangle$ are found using the expectation of (30) over appropriate entries of $\mathbf{H}$. Using (31) and (24), the total average BER can be easily obtained.

\section{EQuivalence of QR AND Regular V-BLAST}

Using an QR decomposition instead of the interference nulling procedure of the traditional V-BLAST algorithm has been proposed in [12] to reduce computational complexity of the algorithm. In this section, we prove that the QR-decomposition based BLAST is identical to the regular (i.e., using projections to null out the inter-stream interference) V-BLAST both in terms of performance and basic processing steps. In fact, the regular BLAST does perform QR decomposition of the channel matrix in an implicit way.

The QR based BLAST operates as follows. Consider a QR decomposition of the channel matrix,

$$
\mathbf{H}=\mathbf{Q R}
$$

where $\mathbf{Q}$ is semi-unitary, $\mathbf{Q}^{+} \mathbf{Q}=\mathbf{I}$, and $\mathbf{R}$ is lowertriangular, $r_{i j}=0$ for $j>i$. Left-multiplying (1) by $\mathbf{Q}^{+}$, one obtains,

$$
\mathbf{r}^{\prime}=\mathbf{Q r}=\mathbf{R s}+\xi^{\prime}
$$

where $\xi^{\prime}=\mathbf{Q} \xi$. Since $\mathbf{R}$ is lower-triangular, the interference from yet to be detected symbols is eliminated,

$$
r_{i}^{\prime}=\sum_{j=1}^{i} r_{i j} s_{j}+\xi_{i}^{\prime}
$$

On the other hand, consider the regular V-BLAST. The normalized weight matrix $\mathbf{W}$ is semi-unitary, and, hence, leftmultiplying $\mathbf{H}$ by $\mathbf{W}^{+}$yields

$$
\mathbf{W}^{+} \mathbf{H}=\mathbf{T}
$$

where $\mathbf{T}$ is lower-triangular. This is also a $\mathrm{QR}$ decomposition of $\mathbf{H}$. Since QR decomposition is unique [7] (up to an ordering), we conclude that $\mathbf{W}=\mathbf{Q}$ and $\mathbf{R}=\mathbf{T}$ (if the same ordering is used in both cases). Hence, two algorithms essentially perform the same processing and, hence, the performance is the same. For an i.i.d. Rayleigh channel, the entries of $\mathbf{H}$ are i.i.d. complex Gaussian, $h_{i j} \sim C N(\mathbf{0}, \mathbf{I})$, and 
the QR decomposition in (32) has the following properties [7]: 1) $\left.\left.r_{i i} \sim \chi_{2(n-m+i)}^{2}, 2\right) r_{i j} \sim C N(0,1), i>j, 3\right)$ all the elements of $\mathbf{R}$ are independent of each other, 4) the elements of $\mathbf{Q}$ and $\mathbf{R}$ are independently distributed and, hence, the noise in (33) is independent of the signal term Rs; furthermore, $\xi^{\prime} \sim \xi \sim C N\left(\mathbf{0}, \sigma_{0}^{2} \mathbf{I}\right)$. These properties are identical to the results obtained above using detailed analysis from the signal processing perspective (the latter gives insight normally not available if the multivariate statistical results are adopted in the final form). This provides an additional validation of our results.

\section{Monte-Carlo Simulations}

In order to verify the analytical results above, extensive Monte-Carlo (MC) simulations have been undertaken. Specifically, the Rayleigh i.i.d. fading channel and BPSK modulation demodulated coherently have been used. First, the instantaneous BER expressions have been validated. No statistically-significant difference between analytical and $\mathrm{MC}$ results have been found for conditional (without error propagation) and unconditional (with error propagation) BER. Secondly, the average BER expressions have been extensively validated. Some of the results are shown in Fig. 1-2.

Fig. 1 shows Monte-Carlo simulated average BER/BLER/TBER and the exact analytical results for $2 \times 2$ systems (for other system orders, similar results have been obtained and not shown here). Good agreement is obvious. $1^{\text {st }}$ step BER dominates the BLER for high SNR $(>5 \mathrm{~dB})$. Note that the error propagation has significant effect on the $2^{\text {nd }}$ step $\mathrm{BER}$, as comparison to the "no error propagation" BER demonstrates (as (23) indicates, the error propagation results in the $2^{\text {nd }}$ step diversity order being equal to one rather than two).

Comparison of the exact and approximate average BLER/TBER expressions of the $2 \times 2$ system indicates that the average BLER is well approximated by the $1^{\text {st }}$ step average BER for $\gamma_{0} \geq 5 d B$ (i.e. eq. 27). The accuracy of the TBER approximation (eq. 25) is a bit worse but still acceptable. The small inaccuracy is due to the fact that (25) ignores the error propagation, which demonstrates that the effect of error propagation on the total average BER is indeed small. Including it results in a better approximation,

$$
\bar{P}_{e t} \approx \bar{P}_{e 1}\left(1+\bar{P}_{21}\right) / 2 \approx 1 /\left(7 \gamma_{0}\right)
$$

Comparing (36) to (25), which ignores the error propagation, one concludes that the effect of error propagation on the average total BER is to increase it by about $20 \%$, which is indeed small (contrary to a widely-accepted viewpoint that the error propagation has a dramatically negative effect on VBLAST BER). On the contrary, the $2^{\text {nd }}$ step average BER is affected by the error propagation in a dramatic way: the diversity order decreases from 2 without error propagation to 1 with it (see Fig. 1). Fortunately, since the $1^{\text {st }}$ step BER is dominant, this does not affect significantly the total BER.

Fig. 2 gives the average step BER of $4 \times 4$ system along with the average BLER/TBER. Based on these numerical

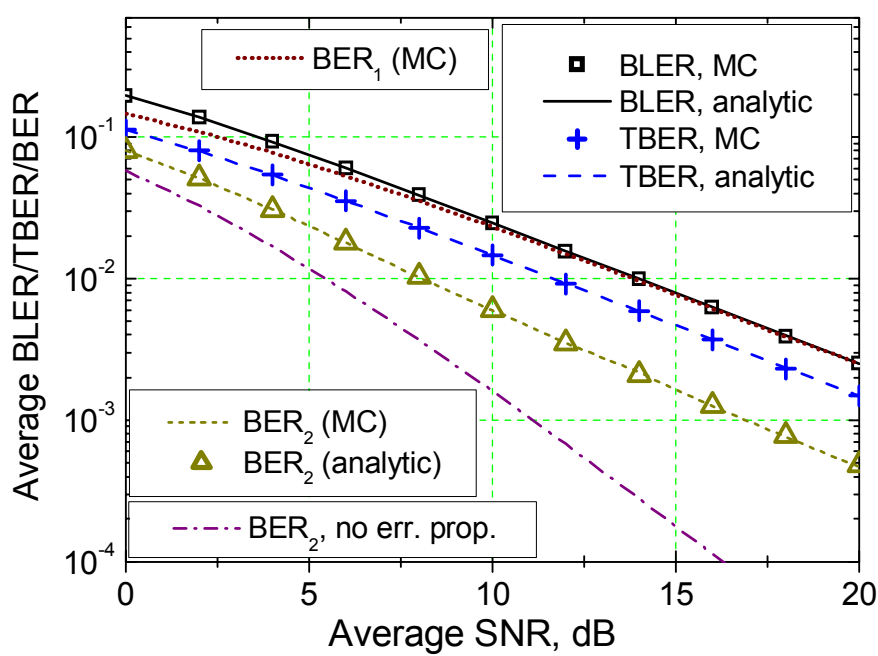

Fig. 1. Average BLER/TBER/BER of 2x2 V-BLAST

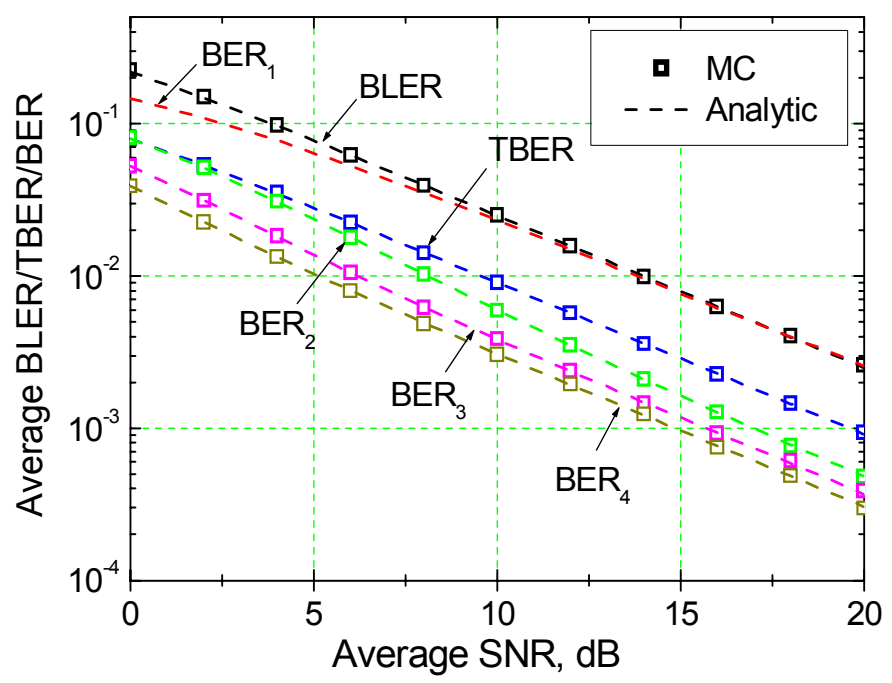

Fig. 2. Average BLER/TBER/BER of $4 \mathrm{x} 4 \mathrm{~V}$-BLAST

results, one observes that the average step BER, despite of the error propagation, decreases with step number,

$$
\bar{P}_{u(i+1)}<\bar{P}_{u i}
$$

According to the analysis above, we attribute this to the fact that (i) the probability of error propagation is less than 1 $(\approx 1 / 5$ for $2 \times 2$ system) and (ii) the conditional BER at higher steps has higher diversity order (see (22) for the case of $2 \times 2$ system). Clearly, the average step BER of $n \times n$ system can be well approximated by

$$
\bar{P}_{u i} \approx a_{i} / \gamma_{0}
$$

where $a_{i}$ are some constants, $a_{i+1}<a_{i}$. We conjecture that $\lim _{i \rightarrow \infty} a_{i}=0$. The approximation in (38) also applies to BLER and TBER. Additionally, one observes that, for high 
SNR, $\bar{P}_{\underline{e t}} \approx \bar{P}_{B} / m \approx \bar{P}_{e 1} / m$. Based on this, we conjecture that $\lim _{n \rightarrow \infty} \bar{P}_{e t}=0$ for nxn system, which demonstrates that VBLAST is asymptotically optimum space-time architecture that achieves the MIMO capacity at arbitrary low BER, even without optimal ordering. Comparing Fig. 2 to Fig. 1, one concludes that the average BER at given step does not depend on whether higher steps are presents or not (for example, $\overline{P_{e 1}}$ is the same for $2 \times 2,3 \times 3$ and $4 \times 4$ systems), which also follows from (31). This conclusion is not entirely trivial as step 1 processing implies projecting out the sub-space spanned by $\left[\mathbf{h}_{2} \mathbf{h}_{3} \ldots \mathbf{h}_{m}\right]$ and, hence, depends on the presence of higherorder steps.

\section{REFERENCES}

[1] G.J. Foschini et al, Analysis and Performance of Some Basic Space-Time Architectures, IEEE Journal Selected Areas Comm., v. 21, N. 3, pp. 281-320, April 2003.

[2] G.J Foschini, 'Layered space-time architecture for wireless communication in a fading environment when using multiple antennas', Bell Lab. Tech. J., vol. 1, N. 2, pp. 41-59, 1996.

[3] G.J Foschini et al, Simplified Processing for High Spectral Efficiency Wireless Communication Employing Multi-Element Arrays, IEEE Journal on Selected Areas in Communications, v. 17, N. 11, pp. 1841-1852, Nov. 1999.
[4] S. Loyka, V-BLAST Outage Probability: Analytical Analysis, IEEE Vehicular Technology Conference, 24-28 September 2002, Vancouver, Canada.

[5] S. Loyka, F. Gagnon, Performance Analysis of the V-BLAST Algorithm: an Analytical Approach, IEEE Trans. Wireless Comm., v. 3, No. 4, pp. 1326-1337, July 2004.

[6] S. Loyka, F. Gagnon, Analytical Framework for Outage and BER Analysis of the V-BLAST Algorithm, International Zurich Seminar on Communications, Feb. 18-20 2004, ETH Zurich, Switzerland

[7] T.W. Anderson, An Introduction to Multivariate Analysis (3rd Ed.), Wiley, 2003.

[8] H.L. Van Trees, Optimum Array Processing, Wiley, 2002.

[9] W.C. Jakes Jr., Microwave Mobile Communication. New York: Wiley, 1974

[10] J.G. Proakis, Digital Communications, McGraw Hill, Boston, 2001.

[11] S. Verdu, Multiuser Detection, Cambridge University Press, 1998.

[12] D. Wubben et al, Efficient Algorithm for Decoding Layered Space-Time Codes, Electronics Letters, v. 37, N. 22, pp. 1348-1350, Oct. 2001.

[13] S. Loyka, F. Gagnon, V-BLAST without Optimal Ordering: Analytical Performance Evaluation for Rayleigh Fading Channels, IEEE Trans. Comm., submitted, 2004. 